with gout in normal and overweight categories were had higher risk vs. controls with FRS-CHD score of $8.86 \pm 8.05$ vs. $6.53 \pm 6.41(p=0.03)$ and $9.37 \pm 7.72$ vs. $7.89 \pm 5.58(p=0.01)$, respectively. No differences for 2013 ACC/AHA in global and BMI-stratified comparisons. Proportion of high-risk subjects were similar in both groups regardless of BMI category: for FRS-CHD 7.5 vs $4.7 \%(p=0.06)$ and 2013 ACC/AHA with 21.5 vs. $17.8 \%(p=0.14)$

Conclusion: Normal BMI or overweight Mexican patients with gout may be at higher 10-year estimated cardiovascular risk compared to matched age, gender and BMI healthy controls. Obese gout subjects are at high risk with non-statistical significance scores for FRS-CHD or 2013 ACC/AHA compared to controls. REFERENCES:

[1] Acosta-Cázares B, Escobedo-de la Peña J. High burden of cardiovascular disease risk factors in Mexico: An epidemic of ischemic heart disease that may be on its way?. American Heart Journal. 2010;160:230-6.

[2] Sánchez Rodríguez A, Moreno-Del Castillo C, Prado Anaya CA et al. ESTIMATED 10-YEAR CARDIOVASCULAR RISK WITH FRAMINGHAM RISK AND 2013 ACC/AHA IN GOUT AND HEALTHY MEXICAN SUBJECTS: A CASE-CONTROL STUDY WITH A PROPENSITY SCORE-MATCHED ANALYSIS J Clin Rheumatol: 2019;25:S1-S96.

[3] Bevis M, Blagojevic-Bucknall M, Mallen C, Hider S, Roddy E. Comorbidity cluster in people with gout: an observational cohort study with linked medial record review. Rheumatology (Oxford). 2018 Apr 17.

[4] Andrés M, Bernal JA, Sivera F, Quilis N, Carmona L, Vela P, Pascual E. Cardiovascular risk of patients with gout seen at rheumatology clinics following a structured assessment. Ann Rheum Dis. 2017;76:1263-8.

Disclosure of Interests: None declared.

DOI: 10.1136/annrheumdis-2021-eular.4226

\section{POS1150 ANATOMICAL LOCATIONS AND CORRELATES OF CALCIUM PYROPHOSPHATE CRYSTAL DEPOSITS OF THE SPINE - PATHOLOGIC EXAMINATION OF 77 SURGICAL CASES}

J. Dau ${ }^{1}$, G. Ho ${ }^{2}$, H. Choi ${ }^{1}$, J. Schwab ${ }^{3}$, M. Kohler ${ }^{1} .{ }^{1}$ Massachusetts General Hospital, Division of Rheumatology, Allergy, and Immunology, Boston, United States of America; ${ }^{2}$ New York University Langone Health, Division of Rheumatology, New York City, United States of America; ${ }^{3}$ Massachusetts General Hospital, Department of Orthopaedic Surgery, Boston, United States of America

Background: Spinal involvement in calcium pyrophosphate deposition disease (CPPD) is thought to be a rare occurrence and is seen infrequently as crowned dens syndrome. Furthermore, data on anatomical locations and correlates of calcium pyrophosphate (CPP) deposits in spinal CPPD are scarce.

Objectives: To describe the anatomical locations and correlates of pathologically confirmed CPPD of the spine.

Methods: Consecutive patients with spinal CPPD were identified via retrospective chart review of individuals who underwent spine surgery for intractable chronic neck or back pain at Massachusetts General Hospital between 2009 and 2014. These deposits and surrounding anatomical structures were surgically resected and confirmed to have calcium pyrophosphate deposition upon pathologic review. We reviewed musculoskeletal imaging (CT, MRI, XR) and laboratory data from these pathologically confirmed cases.

Results: From April 2009 to August 2014, we identified 77 individuals with pathologically confirmed CPPD of the spine. The mean age was 68 years; 41 (53\%) were female; mean BMI was 28.7. Calcium pyrophosphate (CPP) was grossly identified intraoperatively by the surgeon in 38 cases (50\%), typically as "chalky white deposits" (Figure 1). CPP deposits were seen most frequently in the ligamentum flavum (23\%) and intervertebral disc (23\%), followed by other less common locations (Table 1). Imaging findings in the soft tissue or intervertebral disc suggestive of CPPD were found in 5 cases $(6 \%)$, whereas findings of spinal canal narrowing, facet arthropathy, or ligamentum flavum thickening were eventually correlative with CPP deposits in pathologic specimens. Only 7 ( $9 \%$ ) experienced a prior episode of acute CPP arthritis (pseudogout). Chondrocalcinosis on x-ray was seen in 26 cases $(34 \%)$, most commonly in the wrist and/or knees. Osteoarthritis was present in all spinal imaging, and $65 \%$ had comorbid scoliosis. Laboratory abnormalities associated with secondary causes of CPPD (hypercalcemia, hypomagnesemia, hyperparathyroidism) were not seen with spinal CPPD.

Conclusion: Spinal CPPD may occur more frequently than previously perceived. The ligamentum flavum and intervertebral discs were common anatomical locations for spinal CPPD. Advanced imaging of the spine showed low sensitivity for detecting spinal CPPD. Only a small minority had typical peripheral joint involvement or imaging with peripheral joint chondrocalcinosis. Thus, without pathologic confirmation, the vast majority of cases would remain unidentified. These findings call for the need to seek pathologic confirmation to determine the robust epidemiology and also raise the potential role for preoperative CPPD treatment.
Table 1. Spinal Anatomic Locations of Pathologically Confirmed CPPD

\begin{tabular}{lc}
\hline Spinal Anatomic Location & No. of Sites $(\%)^{\star}$ \\
\hline ligamentum flavum & $29(23)$ \\
Intervetebral Disc & $28(23)$ \\
Other Location & $19(15)$ \\
Posterior Elements & $18(15)$ \\
Facet & $14(11)$ \\
Synovium & $8(6)$ \\
Interspinous Ligament & $3(2)$ \\
Subarticular/Lateral Recess & $2(2)$ \\
Fibrocartilaginous Tissue & $1(1)$ \\
Inner Spine & $1(1)$ \\
Other Ligament & $1(1)$
\end{tabular}

*Some patients had more than one anatomic location where CPP was isolated

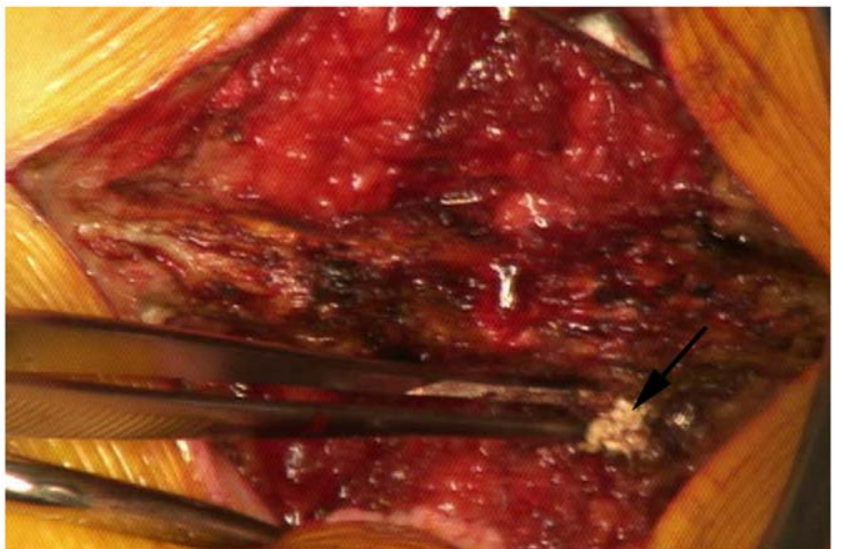

Figure 1. Gross visualization of calcium pyrophosphate deposition (black arrow)

Disclosure of Interests: Jonathan Dau: None declared, Gary Ho: None declared, Hyon Choi Consultant of: Ironwood, Selecta, Horizon, Takeda, Kowa, Vaxart, Grant/research support from: Ironwood, Horizon, Joseph Schwab: None declared, Minna Kohler Speakers bureau: Eli Lily, Consultant of: Novartis. DOI: 10.1136/annrheumdis-2021-eular.4313

\section{Infection-related rheumatic diseases}

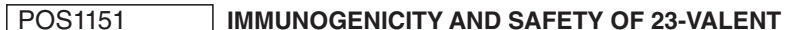 PNEUMOCOCCAL POLYSACCHARIDE VACCINE IN PATIENTS WITH SPONDYLOARTHRITIS.}

N. Muravyeva ${ }^{1}$, B. Belov ${ }^{1}$, M. Baranova ${ }^{1}$, M. Cherkasova ${ }^{2}{ }^{1}$ V.A. Nasonova Research Institute of Rheumatology, Laboratory for Studying Comorbid Infections and Monitoring the Safety of Drug Therapy, Moscow, Russian Federation; ${ }^{2}$ V.A. Nasonova Research Institute of Rheumatology, Laboratory of Immunology and Molecular Biology of Rheumatic Diseases, Moscow, Russian Federation

Background: Currently, for the treatment of patients with spondyloarthritis (SpA) basic anti-inflammatory drugs and biological drugs are widely used to effectively control the activity of the disease. At the same time, the use of these drugs is associated with an increased risk of developing infections, some of which can be prevented by vaccination.

Objectives: The aim of the study was to evaluate the immunogenicity and safety of the 23-valent pneumococcal polysaccharide vaccine (PPV-23) in patients with $\mathrm{SpA}$.

Methods: The study included 42 patients with SpA: 30 men, 12 women, age $22-60$ years, disease duration $14.8 \pm 10.6$ years. Ankylosing spondylitis was diag nosed in 30 patients, psoriatic spondyloarthritis - in 12 patients. Activity of diseases was assessed as high in $78 \%$ of patients (median BASDAI index was 5.3 $[4.1 ; 6.8])$. At the time of inclusion in the study, all patients received nonsteroida anti-inflammatory drugs, 14 patients - methotrexate, 10 - sulfasalazine, 6 - glucocorticoids, 2 - leflunomide, 2 - etanercept, 2 - secukinumab. 15 patients were planned to be prescribed secukinumab, 3 - etanercept, 3 - adalimumab, 1 golimumab. 7 patients had a history of more than 2 cases of lower respiratory tract infections, 2 patients reported a monthly exacerbation of chronic sinusitis, 1 patient - the development of otitis every 2-3 months, requiring the use of antibacterial drugs. PPV-23 was administered in the amount of 1 dose $(0.5 \mathrm{ml})$ subcutaneously into the deltoid muscle against the background of anti-rheumatic therapy. 
The level of antibodies to pneumococcal capsular polysaccharide was determined using the EIA PCP IgG kit (TestLine Clinical Diagnostics s.r.o., Czech Republic) before vaccination, 1, 3 and 12 months after vaccination.

Results: The dynamics of the concentration of antibodies to pneumococcal capsular polysaccharide in patients with SpA is presented in the Table 1.

Table 1. Concentration of pneumococcal antibodies, U/ml, Me [25; 75 percentile]

1 visit (initial) $\quad 2$ visit (after 1 month) 3 visit (after 3 month) 4 visit (after 12 month) $80.0[35.2 ; 154.0] \quad 160.1[73.5 ; 245.7]$ * $214.5[103.2 ; 255.0]$ ** $175.0[120.1 ; 260.1]$ *

${ }^{*} \mathrm{p}=0.01{ }^{* *} \mathrm{p}=0.005$

At 1, 3 and 12 months after vaccination, the concentration of antibodies to pneumococcal capsule polysaccharide was significantly higher compared to the baseline values. In $81 \%$ of patients, vaccination tolerance was good. Reactions at the injection site (pain, swelling and hyperemia of the skin up to $2 \mathrm{~cm}$ in diameter), resolved independently after 1-5 days, were observed in 6 patients. In 2 patients, a severe local reaction was registered in the form of pain in the arm, infiltration and hyperemia of the skin up to 8 and $15 \mathrm{~cm}$ in diameter, respectively, accompanied by low-grade fever in one patient for 2 days, and febrile fever in the other for 3 days. In both cases, these symptoms were completely stopped after administration of paracetamol and antihistamines. Exacerbation of SpA and the emergence of new autoimmune disorders were not detected. During the follow-up period, no patients developed lower respiratory tract infections. Patients suffering from frequent sinusitis and otitis reported the absence of these infections after vaccination.

Conclusion: The obtained data indicate satisfactory immunogenicity and good tolerability of PPV-23 in patients with SpA. Further studies are needed to better assess the immunogenicity and safety of vaccine, as well as to study of the influence of anti-rheumatic therapy on the effectiveness of immunization.

Disclosure of Interests: None declared.

DOI: 10.1136/annrheumdis-2021-eular.505

\section{POS1152 COMORBID INFECTIONS IN PATIENTS WITH SPONDYLOARTHRITIS.}

M. Baranova ${ }^{1}$, N. Muravyeva ${ }^{1}$, B. Belov ${ }^{1} .{ }^{1}$ V.A. Nasonova Research Institute of Rheumatology, Department of Study Comorbid Infection and Monitoring Antirheumatic Therapy, Moscow, Russian Federation

Background: Data on the frequency and structure of comorbid infections $(\mathrm{Cl})$ in spondyloarthritis ( $\mathrm{SpA})$ are few and contradictory.

Objectives: The aim of the study was to study the frequency and structure of $\mathrm{Cl}$ in the inpatient population of SpA patients in the course of a one-moment retrospective study.

Methods: The study included 208 patients with SpA (121 men, 87 women, mean age $39.1 \pm 12.2$ years) who were hospitalized at the V.A. Nasonova Research Institute of Rheumatology. Ankylosing spondylitis was diagnosed in 133 patients, psoriatic arthritis - in 57, spondyloarthritis associated with Crohn's disease - in 1 , undifferentiated spondyloarthritis - in 17. The majority of respondents had higher education $(60.6 \%)$. None of the patients consumed alcohol on a daily basis, 124 patients never smoked. The Charlson comorbidity index, equal to 0 , had 98 respondents, $1-51,2-27,3-15,4-10,5$ or more - 7. Most patients $(\mathrm{n}=168)$ received nonsteroidal anti-inflammatory drugs (NSAIDs), as well as glucocorticoids-GC (average duration of administration $239.5 \pm 65.8$ months), methotrexate-MT $(32.4 \pm 46.2)$, sulfasalazine $(21.0 \pm 32.1)$, leflunomide $(24.0 \pm 46.6)$, biological drugs - TNF-a inhibitors (21.5 \pm 23.3 ), inhibitors of interleukin (ilL)-12/23 (9.0 \pm 5.2$)$, ilL-17 (11.0 \pm 9.3$)$. Patients were interviewed by a research doctor with the completion of a unified questionnaire, additional data were obtained from medical documentation.

Results: Leading in the structure of $\mathrm{Cl}$ in patients with $\mathrm{SpA}$ were respiratory tract infections: acute nasopharyngitis $(n=168)$, tonsillitis $(74)$, acute bronchitis (34), sinusitis (33), pneumonia (29, including 9 cases by the virus SARSCoV-2), influenza (31), tuberculosis-TB (5, including 2 cases on infliximab therapy, which was the reason for withdrawal of the drug). Infectious diseases of other localization include herpes-viral infections $(n=109)$, mycoses (51), urinary tract infections (47), conjunctivitis and blepharitis (37), otitis (25), genital infections (15), skin infections (14), intestinal infections (13), COVID19 without lung damage (19), nervous system infections (2), bone infections (2), chronic hepatitis B (1), chronic hepatitis C (1), HIV infection (1), rubella in adulthood (1), measles in adulthood (1). $20.2 \%$ of patients reported more frequent development of $\mathrm{Cl}$ after the onset of the of SpA: 35 of them received immunosuppressive therapy, 7 - monotherapy of NSAIDs. In SpA patients receiving immunosuppressive drugs, there was an increase in the frequency of acute nasopharyngitis (more often 3 times a year), sinusitis, acute bronchitis, pneumonia and herpes-viral infections, in particular herpes zoster. $29.8 \%$ of patients reported a more severe course of $\mathrm{Cl}$ against the background of SpA (12 of them did not receive immunosuppressive drugs). Temporary discontinuation of therapy due to the development of $\mathrm{Cl}$ occurred in $26.4 \%$ of patients. At the same time, in 5 patients treated with GC (including in combination with MT, $n=3$ ), the development of furunculosis was the reason for changing the treatment regimen. In one patient, MT therapy was discontinued due to the frequent development of purulent tonsillitis. Exacerbation of $\mathrm{SpA}$ after $\mathrm{Cl}$ was diagnosed in 84 patients (70 of them received immunosuppressive therapy).

Conclusion: The data obtained indicate the important of the problem of $\mathrm{Cl}$ in $\mathrm{SpA}$. Further studies are needed on large samples of patients in order to find significant risk factors for $\mathrm{Cl}$, study their relationship with clinical characteristics and influence on the course of SpA

Disclosure of Interests: None declared.

DOI: 10.1136/annrheumdis-2021-eular.551

\section{POS1153 RHEUMATIC DISEASE ON BIOLOGICAL THERAPY AND RISK OF ACTIVE TUBERCULOSIS}

S. Abdulaziz ${ }^{1}$, S. Attar ${ }^{2}$, W. Bajhammoh ${ }^{3}$, E. A. Sindi ${ }^{1}$, D. M. Ayish ${ }^{4}$,

E. Bakhashwain ${ }^{5}{ }^{1}$ King Fahad Hospital, Medicine, Division of Rheumatology, Jeddah, Saudi Arabia; ${ }^{2}$ King Abdulaziz University, Medicine,Division of Rheumatology, Jeddah, Saudi Arabia; ${ }^{3}$ King Fahad Hospital, Medicine, Division of Infectious Diseases, Jeddah, Saudi Arabia; ${ }^{4}$ Prince Mohammed Bin Nasser Hospital, Medicine, Division of Rheumatology, Gizan, Saudi Arabia; ${ }^{5}$ East Jeddah Hospital, Medicine,Division of Rheumatology, Jeddah, Saudi Arabia

Background: Treatment with biologic therapy has been associated with a high risk of reactivation of latent tuberculosis (TB). Preventive strategies for tuberculosis remain a crucial step before initiating biologics in rheumatic disease.Treatment with biological therapy has been associated with high risk of reactivation of latent tuberculosis (TB). Prevention strategies remain a crucial step before initiating biologics.

Objectives: We aimed to assess the effectiveness of TB screening before the initiation of biologics and the risk of occurrence of active TB among patients with rheumatic diseases on biologic therapies.

The study aimed to access the effectiveness of TB screening recommendations before the initiation of biological therapy and identify the incidence of active TB among these patients.

Methods: We performed a hospital-based retrospective cohort study among rheumatic disease patients on biological therapy in two centers in Jeddah between January 2005 to December 2019. Medical files were retrospectively reviewed for demographics data, baseline screening for TB, use of prophylaxis, information on DMARDs and biological therapies, and outcomes results were collected.

Results: A total of 365 patients were included over a period of 14 years. Two hundred ninety-two (80\%) had Rheumatoid arthritis (RA), $13 \%$ psoriatic arthritis (PSA), $9 \%$ spondyloarthritis (SPA), $2 \%$ SLE, and $4 \%$ others. The mean age was $47.54( \pm 14.2), 311(85 \%)$ were females with a mean duration of disease 8.45 years $( \pm 6.58)$. Hundred forty-nine $(42.3 \%)$ were on steroids. Anti TNFs were prescribed in $213(58.4 \%)$ patients, Non Anti-TNFs 124 (36.6\%) patients, and Jak inhibitors $18(5 \%)$ patients.TB screening was done to all patients except 3 patients (data missing) before commencing biologics. Forty-four (12.1\%) patients had latent TB at baseline and all received chemoprophylaxis with isoniazid before starting biologics. Four patients with active TB were identified (one with Behcet's disease and three with RA). One patient had a reactivation of latent TB and 3 patients developed de novo TB. Three out of four had an infection in the first 6 months of treatment (one on infliximab and two on rituximab) and one case after 1 year of stopping adalimumab. Two cases had pulmonary TB and two others with extrapulmonary TB (pericarditis and brain abscess each). All four patients with active TB were treated with standard anti TB medications. Three had complete resolution of their TB and one died.

Conclusion: Baseline screening has been effectively carried out in our cohort as per recommendations. Physician should be vigilant not only for reactivation of latent TB but occurrence of de novo TB in patients on biological therapy.

\section{REFERENCES:}

[1] Gardam, M. A. et al. Anti-tumour necrosis factor agents and tuberculosis risk: mechanisms of action and clinical management. Lancet. Infect. Dis. 3, 148155, doi:10.1016/s1473-3099(03)00545-0 (2003).

[2] Winthrop, K. L., Yamashita, S., Beekmann, S. E. \& Polgreen, P. M. Mycobacterial and other serious infections in patients receiving anti-tumor necrosis factor and other newly approved biologic therapies: case finding through the Emerging Infections Network. Clin. Infect. Dis. 46, 1738-1740, doi:10.1086/587989 (2008)

[3] Cantini, F., Niccoli, L. \& Goletti, D. Tuberculosis risk in patients treated with non-anti-tumor necrosis factor- $\alpha$ (TNF- $\alpha$ ) targeted biologics and recently licensed TNF- $\alpha$ inhibitors: data from clinical trials and national registries. $J$ Rheumatol. Suppl. 91, 56-64, doi:10.3899/jrheum.140103 (2014). 